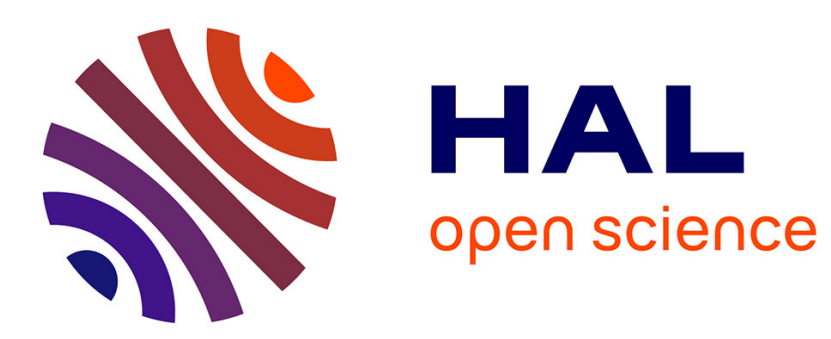

\title{
Etude de diodes Schottky réalisées sous ultra-vide par dépôt d'aluminium ou d'argent sur GaP type $\mathrm{N}$ clivé
}

M. Benkacem, M. Dumas, J.-M. Palau, L. Lassabatère

\section{To cite this version:}

M. Benkacem, M. Dumas, J.-M. Palau, L. Lassabatère. Etude de diodes Schottky réalisées sous ultravide par dépôt d'aluminium ou d'argent sur GaP type N clivé. Revue de Physique Appliquée, 1989, 24 (1), pp.45-50. 10.1051/rphysap:0198900240104500 . jpa-00246027

\section{HAL Id: jpa-00246027 https://hal.science/jpa-00246027}

Submitted on 1 Jan 1989

HAL is a multi-disciplinary open access archive for the deposit and dissemination of scientific research documents, whether they are published or not. The documents may come from teaching and research institutions in France or abroad, or from public or private research centers.
L'archive ouverte pluridisciplinaire HAL, est destinée au dépôt et à la diffusion de documents scientifiques de niveau recherche, publiés ou non, émanant des établissements d'enseignement et de recherche français ou étrangers, des laboratoires publics ou privés. 


\title{
Etude de diodes Schottky réalisées sous ultra-vide par dépôt d'aluminium ou d'argent sur GaP type $\mathbf{N}$ clivé
}

\author{
M. Benkacem, M. Dumas, J. M. Palau et L. Lassabatère \\ Laboratoire d'Etudes des Surfaces, Interfaces et Composants (UA CNRS 040787), Université des Sciences et \\ Techniques du Languedoc, Place Eugène Bataillon, 34060 Montpellier Cedex, France
}

(Reçu le $1^{\text {er }}$ juillet 1988, accepté le 20 septembre 1988)

\begin{abstract}
Résumé. - On présente les résultats de la caractérisation $I(V)$ en direct et $1 / C^{2}(V)$ en inverse de diodes Schottky $\mathrm{Al} / \mathrm{GaP}$ et $\mathrm{Ag} / \mathrm{GaP}$ réalisées sous ultra-vide sur la face (110) clivée d'un barreau de type $\mathrm{n}$. Deux types de surfaces ont été utilisées : les surfaces vierges et les surfaces recuites à $650 \mathrm{~K}$ pendant une heure. Ces surfaces ont préalablement été étudiées à la sonde de Kelvin et par spectroscopie Auger [1,2]. Les diodes ont une barrière $I(V)$ comprise entre 1,1 et $1,2 \mathrm{eV}$. Les caractéristiques $1 / C^{2}(V)$ sont linéaires mais conduisent à des hauteurs de barrière très sensiblement supérieures. De façon générale, le recuit préalable de la surface conduit à des diodes de moins bonne qualité : barrière légèrement diminuée, accroissement du facteur d'idéalité, non-linéarité à bas niveau de courant. Nous proposons une explication basée sur la présence d'états d'interface enfouis, déterminant la hauteur et la forme de la barrière et pouvant participer au transport du courant.

Abstract. - Forward $I(V)$ and reverse $1 / C^{2}(V)$ results obtained for $\mathrm{Al} / \mathrm{GaP}$ and $\mathrm{Ag} / \mathrm{GaP}$ Schottky diodes achieved under ultra-high vacuum condition on cleaved $\mathbf{n}$ type bars are presented. Both unannealed and annealed (650 K, an hour) cleaved (110) surfaces have been used. These surfaces where previously caracterized by work function and AES measurements $[1,2]$. The $I(V)$ Schottky barrier is in the 1.1-1.2 eV range. The barrier height deduced from $1 / C^{2}(V)$ study is greater. Generally, annealed surfaces lead to poorer diodes : decrease of the barrier height, increase of the ideality factor, excess current at low biasing. Results can be explained by considering that buried interface states account for the height and the shape of the barrier and can also contribute to the current transport.
\end{abstract}

\section{Introduction.}

Le développement des techniques d'analyse des surfaces sous ultra-vide au cours des deux dernières décennies, a permis une avancée importante dans la compréhension de l'interaction métal-semiconducteur, en particulier dans le cas des composés III-V. Ces matériaux, outre leur intérêt technologique lié à la largeur de leur bande interdite ou à leur mobilité, peuvent présenter une surface exempte d'état électronique dans la bande interdite : c'est la surface (110) obtenue par clivage [3-5]. Il a ainsi été démontré que l'interaction de ces surfaces avec un métal (ainsi d'ailleurs qu'avec des atomes non métalliques) produit, dès les premiers stades du dépôt, un ancrage du niveau de Fermi qui explique en grande partie la valeur de la barrière $\Phi_{B}$ mesurée dans les diodes Schottky [6-9]. Le phosphure de gallium a suscité, pour ce type d'étude, beaucoup moins d'intérêt que ses homologues GaAs, InP ou
GaSb. Après avoir étudié sa surface clivée et montré que, contrairement aux conclusions de certaines études [10-13], elle ne présente pas de courbure de bande initiale importante $[1,2]$, nous avons réalisé des diodes Schottky par dépôt sous ultravide d'aluminium ou d'argent. Nous présentons dans cet article les résultats que nous avons obtenus, en particulier les hauteurs de barrière déduits des caractéristiques $I(V)$ en direct et $1 / C^{2}(V)$ en inverse. D'autres diodes, réalisées sur des surfaces clivées préalablement recuites à $650 \mathrm{~K}$, ont aussi été réalisées et leurs caractéristiques sont comparées aux premières.

\section{Conditions expérimentales.}

Les surfaces (110) sont obtenues par clivage sous ultra-vide $\left(10^{-10}\right.$ torr) de barreaux de GaP type $\mathrm{n}$ de section $4 \times 8 \mathrm{~mm}$, de dopage $5 \times 10^{15} \mathrm{~cm}^{-3}$ (ou $\left.8 \times 10^{17} \mathrm{~cm}^{-3}\right)$. Le barreau de GaP est entièrement enfermé dans un manchon en cuivre qui peut être 
chauffé ou refroidi. L'évaporation métallique est aussi réalisée dans des conditions d'ultra-vide et dans la même enceinte à partir de creusets chauffés par effet Joule, en carbone pour l'argent, en nitrure de bore pour l'aluminium. Le flux d'évaporation, confiné dans des parois refroidies à l'azote liquide, est contrôlé et régulé par un spectromètre de masse. Ainsi les premières couches métalliques sont déposées à une vitesse inférieure à 3 monocouches par minute et sous un vide résiduel meilleur que $10^{-9}$ torr. Le dépôt s'effectue au travers d'un masque percé de trous de $1 \mathrm{~mm}$ de diamètre. L'épaisseur finale des plots, 2000 à $3000 \AA$, est obtenue par un accroissement progressif de la vitesse d'évaporation au-delà de $100 \AA /$ min. Les diodes sont séparées du barreau par clivage d'une tranche de $2 \mathrm{~mm}$ d'épaisseur. Elles sont caractérisées à l'extérieur du bâti à la température ambiante. Un contact ohmique est réalisé sur toute la face arrière de la tranche par diffusion par étincellage d'or-germanium, technique qui ne produit aucun échauffement de l'échantillon et ne nécessite pas de recuit. La tranche est ensuite collée à la laque d'argent sur un support métallique. Le contact côté plot est pris par pression. La barrière de Schottky $\Phi_{\mathrm{BI}}$ et le potentiel de diffusion $V_{\mathrm{D}}$ sont déterminés de façon habituelle selon les relations classiques [14] où $V$ est la différence de potentiel entre le métal et la limite de la zone de charge d'espace :

$$
\begin{gathered}
I=A^{*} S T^{2} \exp \left(\frac{-\Phi_{\mathrm{BI}}}{k T}\right)\left[\exp \left(\frac{e V}{n k T}\right)-1\right] \\
I / C^{2}=\frac{2}{S^{2} e \varepsilon N_{\mathrm{d}}}\left[V_{\mathrm{D}}-V-\frac{k T}{e}\right] .
\end{gathered}
$$

Nous noterons

$$
\Phi_{\mathrm{BC}}=-e V_{\mathrm{D}}+\left(E_{\mathrm{C}}-E_{\mathrm{F}}\right)_{\mathrm{volume}} .
$$

\section{Résultats et discussion.}

Les contacts $\mathrm{GaP} / \mathrm{Al}$ et $\mathrm{GaP} / \mathrm{Ag}$ sont redresseurs. Des exemples des caractéristiques directes obtenues sont donnés figure 1 . On remarque que si la nature du métal se manifeste de façon modeste dans le cas des surfaces non recuites, elle a un effet important si la surface a subi un recuit préalable. Certaines de ces caractéristiques présentent à bas niveau de courant une distorsion importante par rapport au modèle thermoïonique rappelé par l'équation (1). Ce type
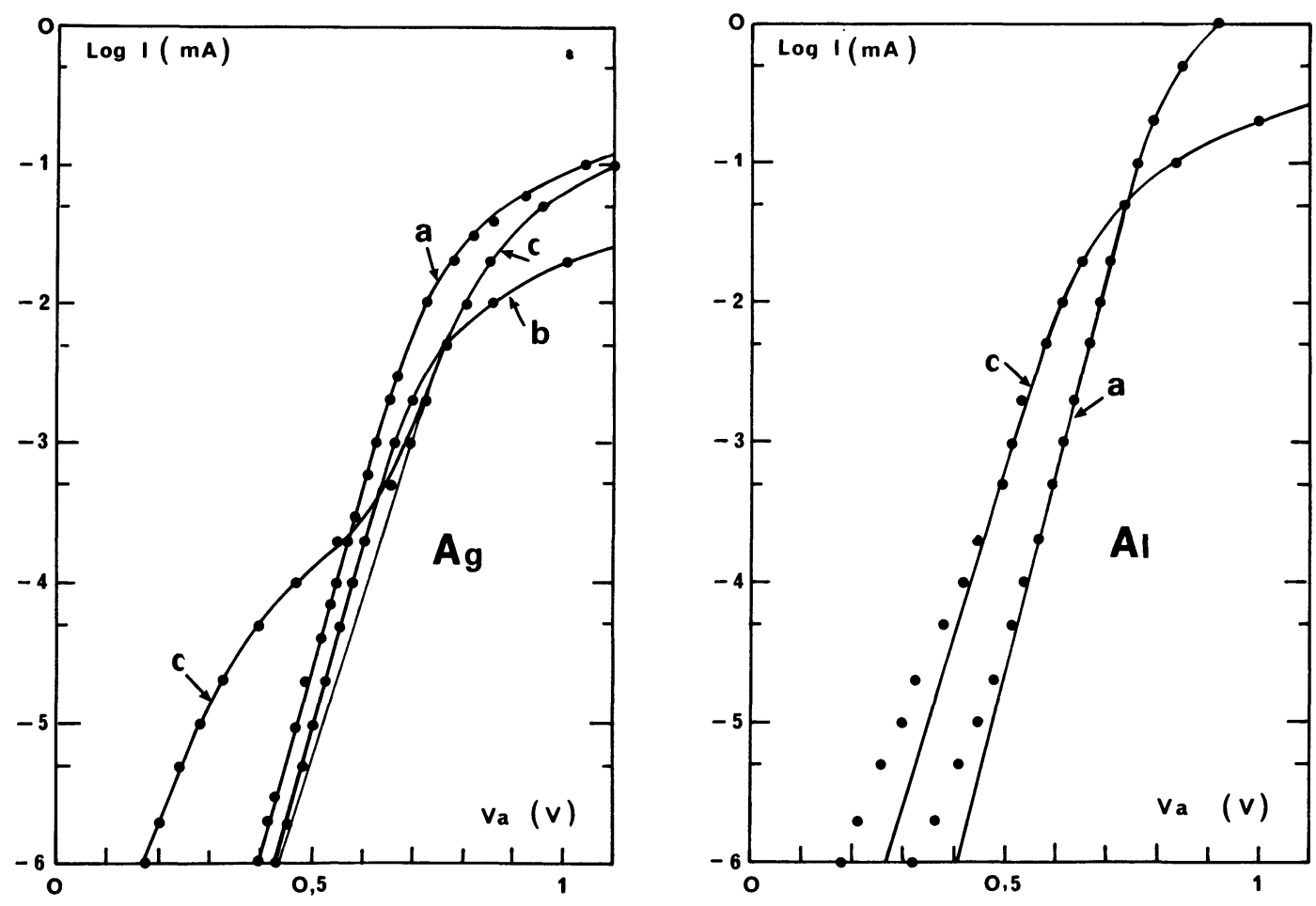

Fig. 1. - Exemples de caractéristiques $I(V)$ directes obtenues après dépôt d'argent ou d'aluminium sur du GaP type $n$ clivé de dopage $5 \times 10^{15} \mathrm{~cm}^{-3}$. a) Surface clivée ; b) Surface clivée recuite à $550 \mathrm{~K}$; c) Surface clivée recuite à $650 \mathrm{~K}$. Les courbes en trait continu sont un ajustement des points expérimentaux suivant l'équation (1) ou suivant un modèle à deux diodes en parallèle [15] ( $\mathrm{Ag}$, courbe $\mathrm{c})$.

[Examples of forward $I(V)$ for silver or aluminium deposited on cleaved $n$ type GaP $5 \times 10^{15} \mathrm{~cm}^{-3}$ doped. a) Cleaved surface ; b) Cleaved surface annealed at $550 \mathrm{~K}$; c) Cleaved surface annealed at $650 \mathrm{~K}$. Continuous curves are a fitting of experimental points using equation (1) or a two parallel diodes model [15] (Ag, c curve).] 
de distorsion déjà signalé par d'autres auteurs sur d'autres matériaux [14-16] se manifeste par l'existence d' « extra courants » dont la croissance avec la tension est rapidement limitée. Ainsi pour déterminer la hauteur de barrière $\Phi_{\mathrm{BI}}$, nous avons ajusté l'équation (1) sur la partie supérieure des courbes expérimentales. Est inclus dans cet ajustement l'effet d'une résistance série $R_{\mathrm{S}}$ qui réduit la tension $V$ aux bornes de la jonction par rapport à la tension appliquée $V_{\mathrm{A}}: V=V_{\mathrm{A}}-R_{\mathrm{S}} I$. L'ajustement est réalisé par un programme de récurrence sur les paramètres $n, R_{\mathrm{S}}$ et $\Phi_{\mathrm{BI}}$. Les valeurs moyennes des paramètres $n$ et $\Phi_{\mathrm{BI}}$ ainsi calculées sont résumées dans le tableau I qui fait aussi apparaitre la dispersion des résultats pourtant obtenus sur un même clivage. L'origine la plus probable de cette dispersion étant la présence d'une densité non uniforme de défauts de clivage, on constate déjà que l'état de la surface avant dépôt a un effet sensible sur les paramètres des diodes. Le tableau I donne aussi la barrière déduite des courbes $1 / C^{2}(V)$, courbes dont l'exploitation suivant l'équation (2) ne présente pas de difficulté car elles présentent une bonne linéarité et leur pente donne correctement le dopage du barreau.

3.1 SurfaCes CLIVÉES VIERGES. - Les valeurs de $\Phi_{\mathrm{BI}}$ obtenues pour l'argent et l'aluminium sont très voisines. Cependant la structure électrique du contact est très certainement différente comme l'indique la différence sensible qui apparaît sur la valeur du facteur d'idéalité et sur la barrière $\Phi_{\mathrm{BC}}$ déduite des études $C(V)$. Si dans le cas de l'aluminium, la différence entre les deux mesures $\Phi_{\text {BI }}$ et $\Phi_{\text {BC }}$ de la barrière de Schottky reste de l'ordre de $100 \mathrm{meV}$ et peut donc être interprétée de façon classique par l'effet de force image ou par une transparence du sommet de la barrière due à un effet tunnel assisté par le champ [14], elle est beaucoup trop importante dans le cas de l'argent. L'interprétation de ce phénomène réside très certainement dans l'explication suivante : le sommet de la barrière est enfoui sous l'interface comme indiqué figure 2 , et de ce fait la barrière $\Phi_{\mathrm{BC}}$ qui est l'extrapolation de la partie parabolique de la zone de charge d'espace est plus grande que $\Phi_{\mathrm{BI}}$. Le profil de la barrière étant déterminé au voisinage de l'interface par des états électroniques [17], ce profil est susceptible de varier sous l'effet de la polarisation appliquée. Cet effet peut être représenté de façon simplifiée par la dépendance suivante : $\Phi_{\mathrm{BI}}=\Phi_{\mathrm{BI} 0}+\alpha \mathrm{eV}$ où le coefficient $\alpha$ est nécessairement positif. Cette simple simulation montre qu'une interprétation de la caractéristique $I(V)$ d'une telle diode suivant l'équation (1) donnera une barrière $\Phi_{\text {BI0 }}$ mais un facteur d'idéalité $n=1 / 1-\alpha$. Ceci montre donc qu'un accroissement du facteur d'idéalité $n$ peut être interprété en terme d'élargissement du sommet de la barrière et donc d'une plus grande dépendance avec la tension appliquée.

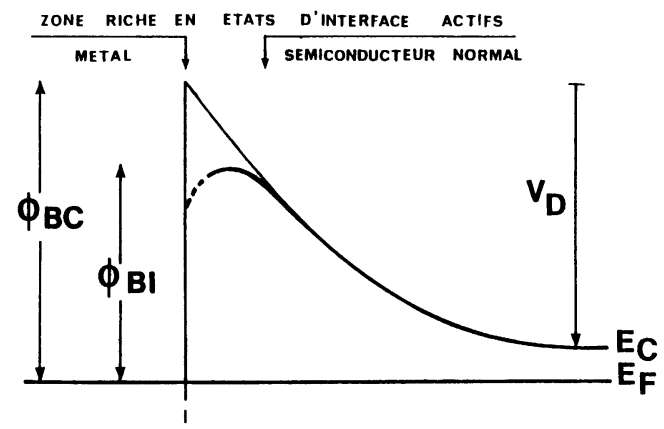

Fig. 2. - Représentation schématique du profil du contact Schottky. La hauteur de barrière $\Phi_{\mathrm{BI}}$ est déterminée par des états chargés [17]. La variation du potentiel au voisinage immédiat du métal peut être beaucoup plus complexe qu'indiqué sur la figure par la courbe en pointillés.

[Schematic diagram of the Schottky contact profile. The barrier height $\Phi_{\text {BI }}$ is fixed by charged states [17]. The potential variation near the metal can be very much complex that shown by the dotted curve of the diagram.]

Tableau I. - Barrière $I(V)$, facteur d'idéalité et barrière $C(V)$ des diodes Schottky réalisées sur GaP type n (la valeur utilisée pour $A^{*}$ est $150 \mathrm{~A} / \mathrm{cm}^{2}$ ).

$[I(V)$ barrier, ideality factor and $C(V)$ barrier of the Schottky diodes achieved on $n$ type GaP (the used value for $A^{*}$ is $\left.150 \mathrm{~A} / \mathrm{cm}^{2}\right)$.]

\begin{tabular}{|c|c|c|c|c|}
\hline \multirow{2}{*}{$\begin{array}{c}\text { GaP } \\
\text { type } n \\
5 \times 10^{15} \mathrm{~cm}^{-3}\end{array}$} & \multicolumn{2}{|c|}{ Surface non recuite } & \multicolumn{2}{c|}{ Surface recuite à $650 \mathrm{~K}$} \\
\cline { 2 - 5 } & $\mathrm{Ag}$ & $\mathrm{Al}$ & $\mathrm{Ag}$ & $\mathrm{Al}$ \\
\hline$\Phi_{\mathrm{BI}}(\mathrm{meV})$ & $1140 \pm 10$ & $1180 \pm 10$ & $1090 \pm 30$ & $1070 \pm 40$ \\
\hline$n$ & $1,30 \pm 0,05$ & $1,15 \pm 0,02$ & $1,50 \pm 0,10$ & $1,25 \pm 0,10$ \\
\hline$\Phi_{\mathrm{BC}}(\mathrm{meV})$ & $1630 \pm 70$ & $1300 \pm 30$ & $1400 \pm 20$ & $1460 \pm 20$ \\
\hline
\end{tabular}


L'élargissement du sommet de la barrière peut être associé à la capacité du métal à établir des liaisons chimiques. En effet il est admis que la réactivité chimique de l'aluminium est plus grande que celle de l'argent. C'est parce que l'interdiffusion métal-semiconducteur serait plus forte dans le cas de l'argent que, le sommet de la barrière étant large et très enfoui, $n$ et la barrière $\Phi_{\mathrm{BC}}$ sont particulièrement élevés pour ce dernier.

3.2 SuRfACES CLIVÉES RECUITES. - L'examen du tableau I montre que le recuit de la surface avant dépôt a un effet important sur les paramètres caractéristiques des diodes. La barrière $\Phi_{\mathrm{BI}}$ est plus faible, le facteur d'idéalité a augmenté, les barrières $\Phi_{\text {BC }}$ sont devenues voisines pour les deux métaux, mais la différence avec $\Phi_{\text {BI }}$ reste très grande. La figure 1 montre que dans le cas de l'argent les « extra courants » ont augmenté de façon considérable. Cet accroissement ne se produit pas pour un recuit de la surface plus modéré à $550 \mathrm{~K}$.

Ces résultats montrent que l'état de la surface détermine en partie la structure de l'interface métalsemiconducteur. La différence avec la surface non recuite ne peut pas être expliquée par une variation significative de la stœchiométrie des surfaces. En effet leur étude par spectroscopie d'électrons Auger (AES) n'a pas permis de mettre en évidence dans ces conditions de recuit une évaporation de phosphore [2]. Par contre, l'étude de ces surfaces par la sonde de Kelvin montre une évolution très nette du travail de sortie sous l'effet du recuit [2] qui se traduit par une uniformisation de celui-ci, comme cela a déjà été observé pour InP [18] et GaAs [35]. Cette uniformisation ainsi que celle du photovoltage qui lui est associée mettent en évidence la création d'états électroniques accepteurs chargés (au plus $10^{12}$ électrons par $\mathrm{cm}^{-2}$ ) [1] que l'on est tout naturellement conduit à attribuer à des défauts. $\mathrm{Si}$ ces défauts sont des lacunes de phosphore, elles ne peuvent pas être observées en AES dont la sensibilité est insuffisante. Les défauts induits par le recuit sont donc en quantité limitée et leur action se manifeste sur les paramètres indicateurs d'une orthodoxie plus ou moins grande avec la forme de barrière théorique (potentiel parabolique jusqu'au métal). On doit considérer que les modifications portent surtout sur la zone de transition entre le métal parfait et le semiconducteur parfait, zone dont la structure est manifestement dépendante du détail de la manière dont s'est produite l'interaction entre le métal et le semiconducteur. Une explication possible de la modification de la zone de transition peut être dans l'augmentation de la «rugosité » de la surface si, comme c'est le cas pour InP [18], la décomposition thermique de GaP débute sur quelques sites et produit des «cavités». Il en résulterait alors un élargissement considérable de la zone de transition.
Parce que les caractéristiques inverses $1 / C^{2}(V)$ sont encore linéaires, on peut considérer que la largeur de cette zone est encore faible devant celle de la zone de charge d'espace et que l'explication de l'écart entre les barrières $\Phi_{\mathrm{BI}}$ et $\Phi_{\mathrm{BC}}$ est du même type que celle avancée pour les surfaces vierges.

Il est clair que l'explication par la forme de la barrière des résultats sur $n$ et $\Phi_{B}$ ne préjuge pas de l'origine des charges responsables de la distorsion de la couche de potentiel dans la région du sommet de la barrière. Des niveaux profonds initialement présents dans le volume et initialement neutres peuvent, du fait de la courbure des bandes, être localement chargés et participer ainsi à cette distorsion. Mais leur effet étant par nature indépendant du métal ou de l'état de la surface (à barrière égale, ce qui est le cas), ils ne peuvent pas rendre compte des variations observées.

3.3 ORIGINE DES EXTRA COURANTS. - L'origine des déviations par rapport à la théorie standard de l'émission thermoïonique est de façon classique attribuée, soit à des courants de recombinaison facilités par la présence de centres profonds, soit à des courants tunnels dits assistés par le champ, c'està-dire correspondant à des électrons d'énergie thermique un peu inférieure à la barrière $\Phi_{B}$ et pouvant franchir cette dernière dans sa partie étroite [14]. Ces courants tunnels sont naturellement favorisés si on réduit la largeur de la zone de charge d'espace en augmentant le dopage du semiconducteur. Un effet tunnel via les états d'interface a aussi été proposé [19].

Un exemple des caractéristiques $I(V)$ que nous avons obtenues sur des surfaces clivées vierges d'un échantillon de dopage $8 \times 10^{17} \mathrm{~cm}^{-3}$ est donné figure 3. Il montre que les extra courants sont devenus très importants pour les deux métaux. Si on note que la largeur de la zone de charge d'espace a été réduite environ d'un facteur 30 , on est naturellement conduit à penser que l'augmentation des extra courants est due à une augmentation de l'effet tunnel.

Nous pensons que la hauteur de la barrière de Schottky dans les III-V, est déterminée pour l'essentiel par des états électroniques répartis sur plusieurs couches atomiques à l'intérieur du semiconducteur et que le sommet de la barrière se trouve dans cette couche d'états [17]. Les états les plus éloignés du métal, qui ne sont plus en équilibre avec ce dernier, ce qui explique la variation de $\Phi_{B}$ avec la tension appliquée, peuvent servir de relais dans le transport des électrons. Dans cette hypothèse, leur densité et leur distribution dans la direction perpendiculaire à l'interface est aussi un paramètre déterminant de l'importance des extra courants. Ainsi la valeur élevée de ces derniers dans le cas des diodes 


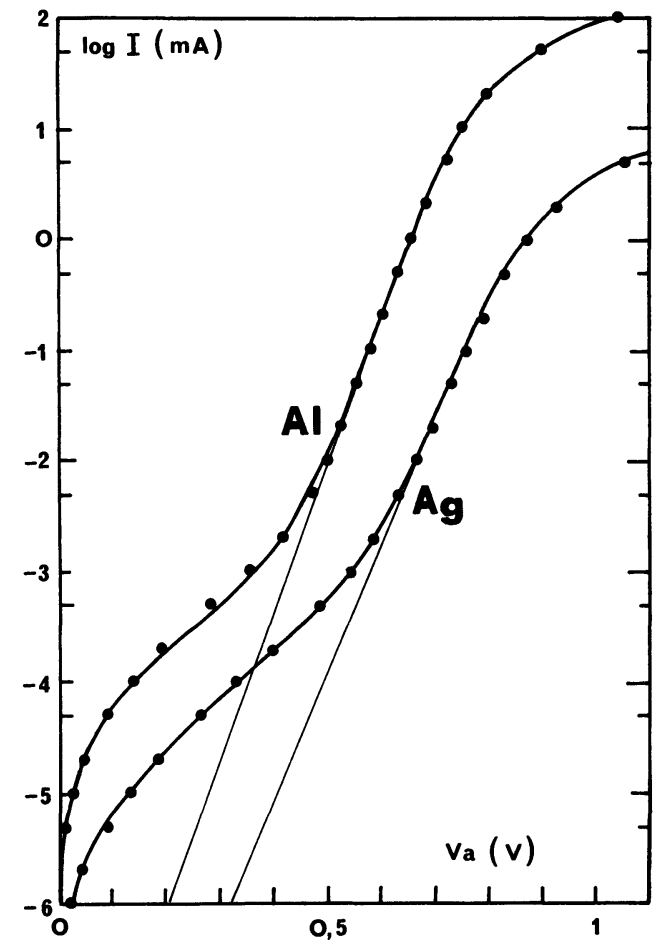

Fig. 3. - Caractéristiques $I(V)$ directes obtenues avec un dopage $n=8 \times 10^{17} \mathrm{~cm}^{-3}$. L'ajustement des points expérimentaux est réalisé par le modèle à deux diodes [15] avec les paramètres suivants : $\mathrm{Ag}: I_{\mathrm{S} 1}=0,18 \mathrm{pA}, n_{1}=$ $1,44, R_{\mathrm{S} 1}=30 \Omega, I_{\mathrm{S} 2}=3,5 \mathrm{nA}, n_{2}=3,73, R_{\mathrm{S} 2}=86 \mathrm{~K} \Omega$. $\mathrm{Al}: I_{\mathrm{S} 1}=2,3 \mathrm{pA}, n_{1}=1,27, R_{\mathrm{S} 1}=2,3 \Omega, I_{\mathrm{S} 2}=48 \mathrm{nA}$, $n_{2}=4,86, R_{\mathrm{S} 2}=2,8 \mathrm{~K} \Omega$.

[Forward $I(V)$ characteristics obtained for $n=8 \times$ $10^{17} \mathrm{~cm}^{-3}$. Experimental points are fitted by the two parallel diodes model [15] using the following parameters : $\mathrm{Ag}: I_{\mathrm{S} 1}=0.18 \mathrm{pA}, n_{1}=1.44, R_{\mathrm{S} 1}=30 \Omega, I_{\mathrm{S} 2}=3.5 \mathrm{nA}$, $n_{2}=3.73, R_{\mathrm{S} 2}=86 \mathrm{~K} \Omega . \quad \mathrm{Al}: I_{\mathrm{S} 1}=2.3 \mathrm{pA}, n_{1}=1.27$, $R_{\mathrm{S} 1}=2.3 \Omega, I_{\mathrm{S} 2}=48 \mathrm{nA}, n_{2}=4.86, R_{\mathrm{S} 2}=2.8 \mathrm{~K} \Omega$.]

$\mathrm{Ag} /$ surfaces recuites pour $\mathrm{GaP}$ peu dopé peut s'expliquer par une densité plus élevée des états enfouis.

L'hypothèse d'une interface non homogène où coexistent plusieurs barrières a été proposée par divers auteurs [20-23]. Plus particulièrement Barret et al. $[15,16]$ ont montré qu'on pouvait ajuster de façon satisfaisante des courbes expérimentales semblables à celles que nous avons obtenues à l'aide d'un modèle à deux diodes en parallèle, la diode ayant la barrière la plus faible occupant une toute petite fraction de l'aire totale du contact et de ce fait présentant une résistance série très élevée. C'est un ajustement de ce type qui a été utilisé pour les figures $1 \mathrm{c}$ et 3 . Bien que l'ajustement soit presque parfait, le modèle à deux diodes ne nous paraît cependant pas approprié car on voit mal pourquoi le changement de dopage accroîtrait l'aire de la diode associée à la barrière la plus faible. La participation directe des états dans les mécanismes de transport du courant nous paraît donc l'explication la plus appropriée. On peut aussi penser que c'est à cause d'une couche d'états plus large que la résistance série observée pour l'argent est, pour les deux dopages utilisés, anormalement élevée alors que pour l'aluminium elle est conforme à celle que l'on peut calculer à partir du dopage du barreau.

\section{Conclusion.}

Les contacts $\mathrm{Ag}$ ou $\mathrm{Al}$ réalisés sur la surface clivée de GaP type $n$ présentent des hauteurs de barrière $\Phi_{B}$ comprises entre 1 et $1,2 \mathrm{eV}$. Ces valeurs sont dans la plage de celles, relatives à divers types de surfaces, que l'on trouve dans les publications [2429]. Bien que les caractéristiques $1 / C^{2}(V)$ soient linéaires, elles donnent des hauteurs de barrières $\Phi_{\mathrm{BC}}$ nettement supérieures à $\Phi_{\mathrm{BI}}$. Les caractéristiques $I(V)$ peuvent présenter à bas niveau des déviations importantes à la théorie thermoïonique standard. Une altération modérée de la surface par simple recuit accentue ces anomalies et dégrade le facteur d'idéalité. Enfin la différence de réactivité chimique des deux matériaux se manifeste sur les caractéristiques des diodes dans le sens d'un comportement moins idéal pour l'argent que pour l'aluminium.

Ces résultats imposent de considérer que le profil du contact réel est assez éloigné de celui du contact idéal et que les caractéristiques de la zone de transition entre le métal et le semiconducteur jouent un rôle déterminant dans le comportement électrique de la diode. Nous soutenons que cette zone est responsable de la valeur de la hauteur de barrière à cause de la forte densité d'états, situés dans la bande interdite, qu'elle contient; que les détails de sa structure en relation avec la façon dont s'est déroulée l'interaction métal-semiconducteur sont à l'origine des variations des paramètres d'un type de diode à l'autre ; qu'elle participe partiellement au courant en rajoutant des composantes non thermoïoniques, vraisemblablement effet tunnel utilisant les états comme relais. En conclusion, le comportement général du contact métal-GaP est semblable à celui que nous avons observé pour $\mathrm{GaAs}$ et InP, à savoir que la position de l'ancrage du niveau de Fermi à l'interface est sensiblement la même que celle observée à la surface, même en l'absence de métal $[1,9$, 30-34]. 


\section{Bibliographie}

[1] Benkacem, M., Dumas, M., Palau, J. M. and LASSABATĖRe, L., Surf. Sci. 201 (1988) L485.

[2] BenKacem, M., Thèse Univ. Sci. Techn. Languedoc, Montpellier (17 nov. 1987).

[3] Spicer, W. E., Pianetta, P., Lindau, I. and Chye, P. W., J. Vac. Sci. Technol. 14-4 (1977) 885.

[4] Palau, J. M., Testemale, E. and lassabatère, L., J. Vac. Sci. Technol. 19-2 (1981) 192.

[5] Ismail, A., Ben Brahim, A., Palau, J. M. and LASSABATĖRE, L., Surf. Sci. 164 (1985) 43.

[6] Spicer, W. E., Lindau, I., Skeath, P. and Su, C. Y., J. Vac. Sci. Technol. 17 (1980) 1019.

[7] Williams, R. H., J. Vac. Sci. Technol. 18 (1981) 929.

[8] Brillson, J. L., Surf. Sci. Rep. 2 -2 (1982).

[9] Lassabatère, Ismail, A., Palau, J. M. and Ben BRAHIM, A., Surf. Sci. 168 (1986) 336.

[10] VAn LaAR, J., HuiJser, A. and Van RoOY, T. L., J. Vac. Sci. Technol. $14-4$ (1977) 894.

[11] Bertoni, C. M., Bisi, O., MANGHI, F. and CALANDRA, C., J. Vac. Sci. Technol. 15 (1978) 1256.

[12] Guichar, G. M., Sebenne, C. A. and Thuault, C. D., J. Vac. Sci. Technol. 16-5 (1979) 1212.

[13] Allen, R. E., HJalmarson, H. P. and Dow, J. D., Surf. Sci. 110 (1981) L625.

[14] Par exemple RHODERICK, E. H., Metal semiconductor contacts, Monograph in Electrical and Electronic Engineering (Clarendon Press, Oxford) 1980.

[15] Chekir, F., Lu, G. N. and BArret, C., Solid State Electron 29-5 (1986) 519.

[16] Barret, C., Lu, G. N. and Neffati, T., Rev. Phys. Appl. 22 (1987) 1485.

[17] Palau, J. M., Ismail, A. and Lassabatère, L., Solid State Electron. 28-5 (1985) 499.

[18] Dumas, M., Benkacem, M., Palau, J. M. et LasSABATÈRE, L., Le Vide, les Couches Minces 43 (1988) 241.
[19] Card, H. C., Solid State Electron. 18 (1975) 881.

[20] Freeouf, J. L. and Woodall, M. J., Appl. Phys. Lett. 39 (1981) 727.

[21] Schneider, M. V., Cho, A. Y., Kolberg, E. and Zirath, H., Appl. Phys. Lett. 43 (1983) 558.

[22] Hanselaer, P. L., Laflere, W. H., VAN Meirhaeghe, R. L. and Cardon, F., J. Appl. Phys. 56 (1984) 2309.

[23] Thomson, R. D. and Tu, K. N., J. Appl. Phys. 53 (1982) 4285.

[24] Nannichi, Y. and Pearson, G. L., Solid State Electron. 12 (1969) 341.

[25] Lei TAN, F., Lee Chung, L. and Chang Chun, Y., Solid State Electron. 22 (1979) 1035.

[26] KusaKa, M., OKamura, M., Hiraoka, N., Hirai, M. and OKAZAKI, S., Surf. Sci. 86 (1979) 841.

[27] Kusaka, M., HiraOKa, N., Hirai, M. and OKAZAKI, S., Surf. Sci. 91 (1980) 264.

[28] LeE, B. W., WANG, D. C., Ni, R. K., XU, G. and Rowe, M., J. Vac. Sci. Technol. 21-2 (1982) 577.

[29] Schwartz, G. P. and Gualtieri, G. J., J. Appl. Phys. 58-12 (1985) 4621.

[30] Ismail, A., Ben Brahim, A., Palau, J. M. and LASSABAtère, L., Surf. Sci. 162 (1985) 195.

[31] Ismail, A., Ben Brahim, A., Palau, J. M. and LASSABATĖRE, L., Vacuum 36-4 (1986) 217.

[32] Ismail, A., Palau, J. M. and Lassabatère, L., $J$. Appl. Phys. 60-5 (1986) 1730.

[33] Ismail, A., Ben Brahim, A., Palau, J. M. and LASSABATĖRe, L., Surf. Sci. 168 (1986) 409.

[34] Ismail, A., Palau, J. M. and lassabatère, L., Surf. Sci. 168 (1986) 386.

[35] Ismail, A., Palau, J. M. and lassabatère, L., Rev. Phys. Appl. 19 (1984) 197. 